\title{
Missing Sensor Data Restoration For Vibration Sensors on A Jet AirCRAfT ENGINE
}

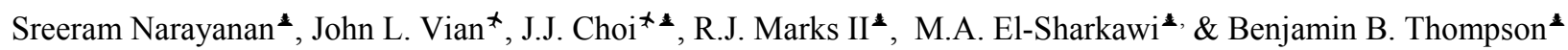

丸 Boeing Phantom Works, The Boeing Company, Seattle, WA 98124

2 Computational Intelligence Applications (CIA) Laboratory, Department of Electrical Engineering, University of Washington, Seattle, WA 98195

\begin{abstract}
Using array historical data, the readings from a sensor array may be shown to contain sufficient redundancy such that the readings from one or more lost sensors may be able to be accurately estimated from those remaining. This interdependency can be established by an neural network encoder. The encoder is also used in the restoration process. In this paper, we give some examples of sensor restoration for vibration sensors on jet engine and computer traffic data.
\end{abstract}

Key Words: neural network, auto-encoder, sensor restoration, auto-associative regression machine.

\section{INTRODUCTION}

In certain cases, sensor readings may be related in such a way as to allow restoration of one or more lost readings from those remaining. Using auto-associative regression machine auto-encoders [Reed \& Marks], missing sensor data (MISED) restoration has been proposed as a method to estimate the readings of failed sensors by recognition or discovery of a constraint placed on the historical readings from the sensor array [Narayanan et al. 2002]. This paper presents example applications of this procedure.

\section{BACKGROUND}

Historical data from a sensor array can be used to train an auto-associative neural network encoder as illustrated in Figure 1. For $N$ sensors, the auto-encoder with $N$ inputs and $N$ outputs, is trained to produce an identity mapping through a degrees-of-freedom bottleneck of $M<N$. If this identity operation can be achieved, there exists a (possibly nonlinear) relationship among the sensor readings. When one or more sensor values are lost, this redundancy can, in certain instances, be used to restore the missing sensor values.

The procedure for MISED is illustrated in Figure 2. The known sensors and initial guesses of the missing sensor values are placed into the auto-encoder. The output of the auto-encoder is subtracted from the distance to specify the error. The error is used to incrementally change the estimated values of the missing sensors and the feedback iteration is repeated. Numerous optimization algorithms can be used to find the missing sensor values that minimize the composite error of the encoder [Narayanan et al. 2002].

\section{JET ENGINE VIBRATION SENSORS}

A finite element emulation of jet engine developed at Boeing Phantom Works is used to illustrate MISED. Four vibration sensors are placed at disperse locations on the engine subjected to imbalances at three locations. Each sensor measures the vibration spectrum in the $x, y$ and $z$ directions. The frequency response consists of 500 points over $50 \mathrm{~Hz}$ each spaced by $0.1 \mathrm{~Hz}$. Example plots of the vibration spectra magnitudes are shown in Figure 3.

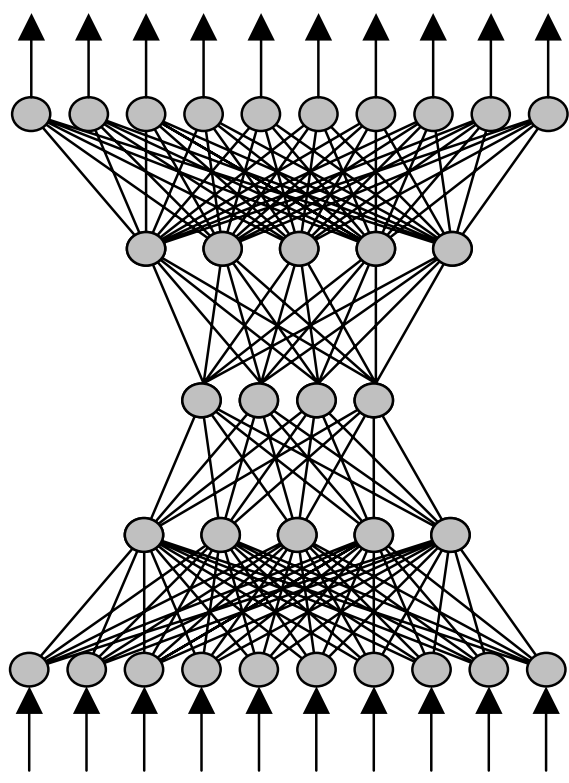

Figure 1: An example 10-5-4-5-10 neural network auto-associative auto-encoder. 


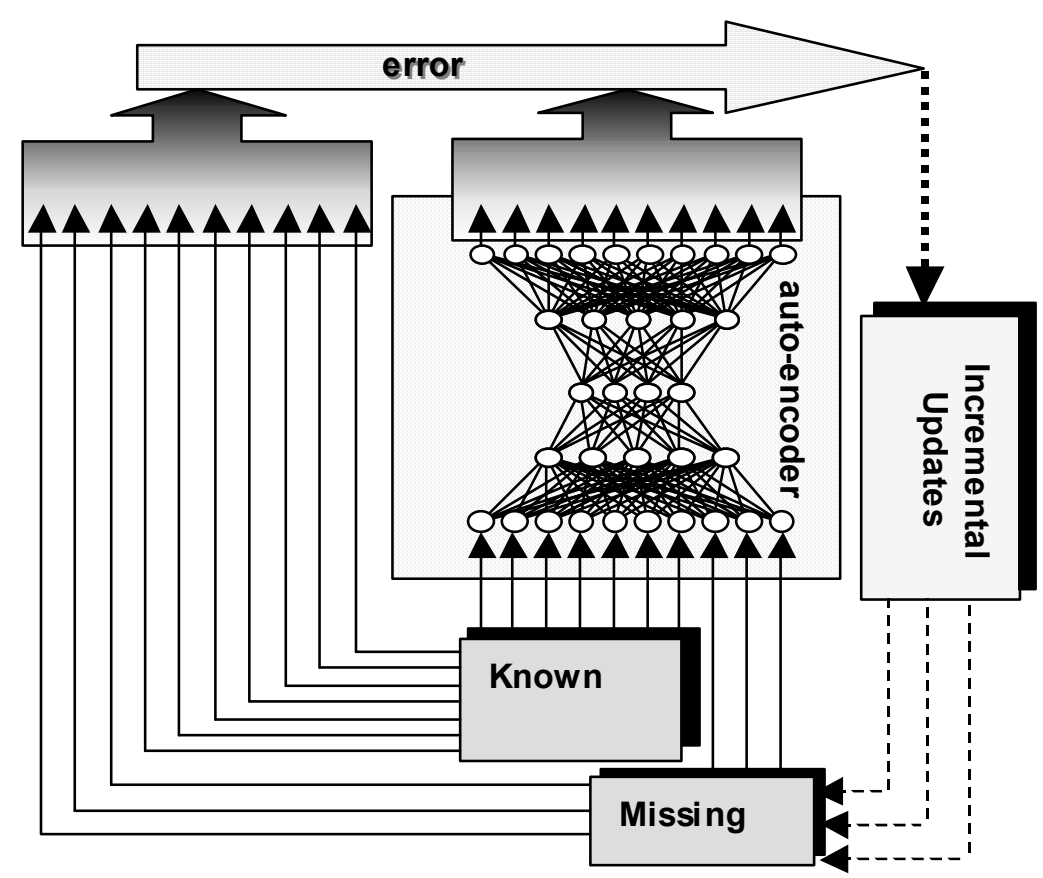

Figure 2: A methodology for restoring missing sensor data using a trained auto-encoder.

training vector is shown at the top of Figure 4. Each eleven numbers correspond to the real part of the reading of sensor number 1 at sequential frequencies. The second eleven numbers correspond to the imaginary part. The next twenty two numbers correspond to the frequency components measured by sensor number two, etc.

The auto-encoder was trained with 5000 sensor readings obtained using the convex data enrichment procedure described by [Thompson et al.]. An additional 500 samples were used for testing. The neural network architecture used gave the lowest test error over those tested. The average RMS error for the testing data was less than $2 \%$ of the maximum. The maximum testing error was $10 \%$ of the maximum.

A second data set consisting of eight sensors placed at different locations and subjected to imbalances at three different locations were also used. Here too each sensor had a frequency response consisting of 500 points spaced over $50 \mathrm{~Hz}$. For the second data set, only the magnitude of each sensor vibration is used. Ten points corresponding to frequencies between $30 \mathrm{~Hz}$ and $31 \mathrm{~Hz}$ was tested. So with 10 points per sensor and eight sensors, there are 80 inputs.

Data enrichment was again used to create 5000

When we try to restore we set the 11 points corresponding to the missing sensor to zero \& try to reconstruct using the trained encoder $\mathrm{NN}$. Using this way we would need about $50 \mathrm{NN}$ 's to train the whole frequency response.

To illustrate MISED, a 88-60-40-60-88 autoencoder was trained with sensor data using standard error back-propagation. Eleven sequential points in the frequency response between $30 \mathrm{~Hz}$ and $31 \mathrm{~Hz}$ was chosen. The neural network was trained on the real and imaginary parts of each point in the frequency spectrum. A sample

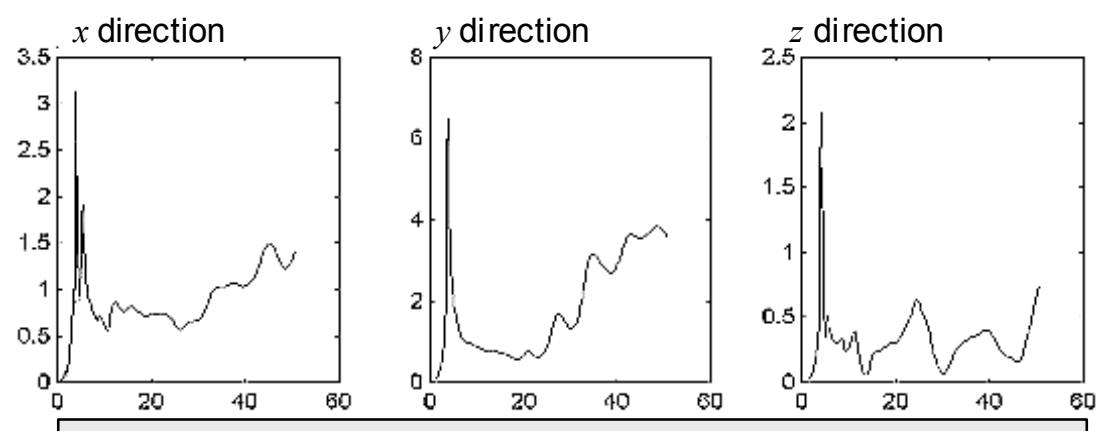

Figure 3: Examp le spectral magnitudes measured by vibration sensors. Frequency is in hertz. training data set and 500 test vectors. For the second data set, auto-encoder neural networks with different architectures were tried to reconstruct the missing sensors. The training and testing RMS errors were less than $3 \%$ of the maximum range of data.

The ability to restore lost sensor measurements was uniformly successful for one and two missing sensors. An example of restoring a single missing sensor is illustrated in Figure 4. Each missing sensor corresponds to 11 lost data points. Shown at the bottom of Figure 4 is the output of the autoencoder for both known and missing sensor readings. The result is graphically indistinguishable from the desired response shown at the top.

For two lost sensors, worst case test results are shown in Figure 5. The maximum error, remarkably, is approximately $5 \%$ of peak. Restoration of three missing sensors is not possible because the number of known values would be reduced to 22 . A necessary condition for MISED to work is that the number of known values must equal or exceed the number of degrees-of-freedom in the bottleneck - in this case 40. The 
Original Sensor Data

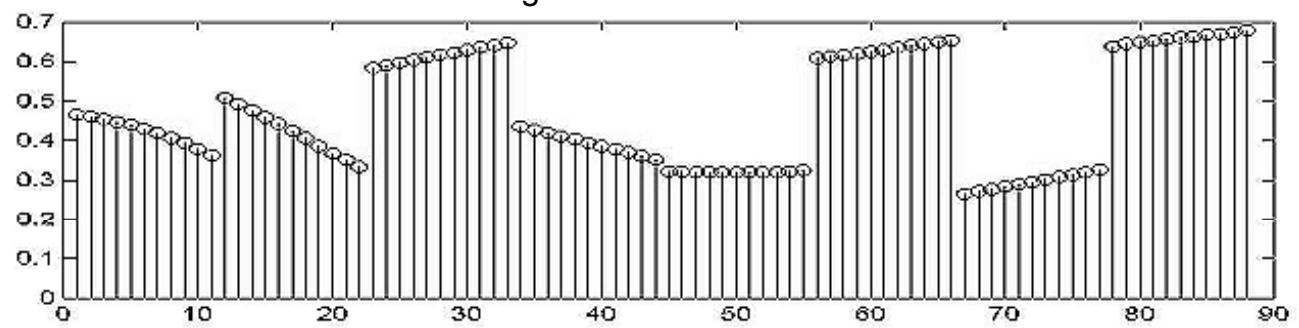

Reconstructed sensor data when sensor 4 fails.

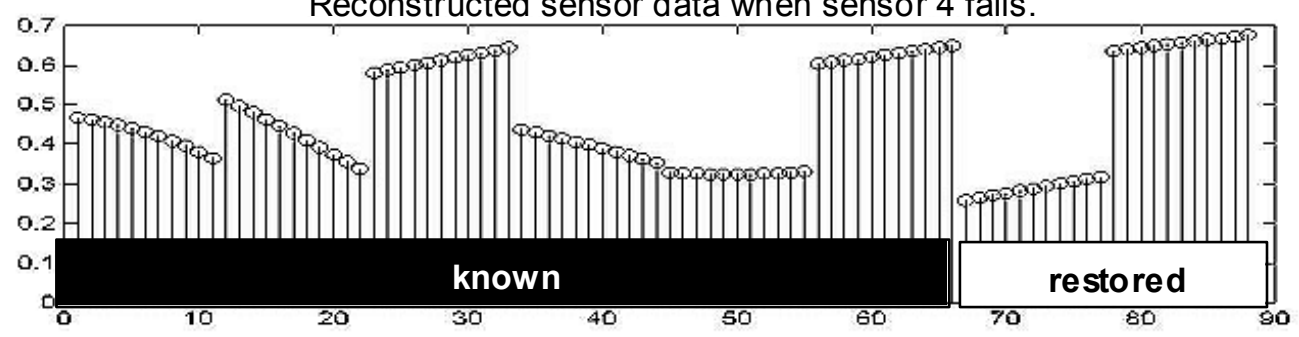

Figure 4: Orig inal (top) and restored missing sensor data.
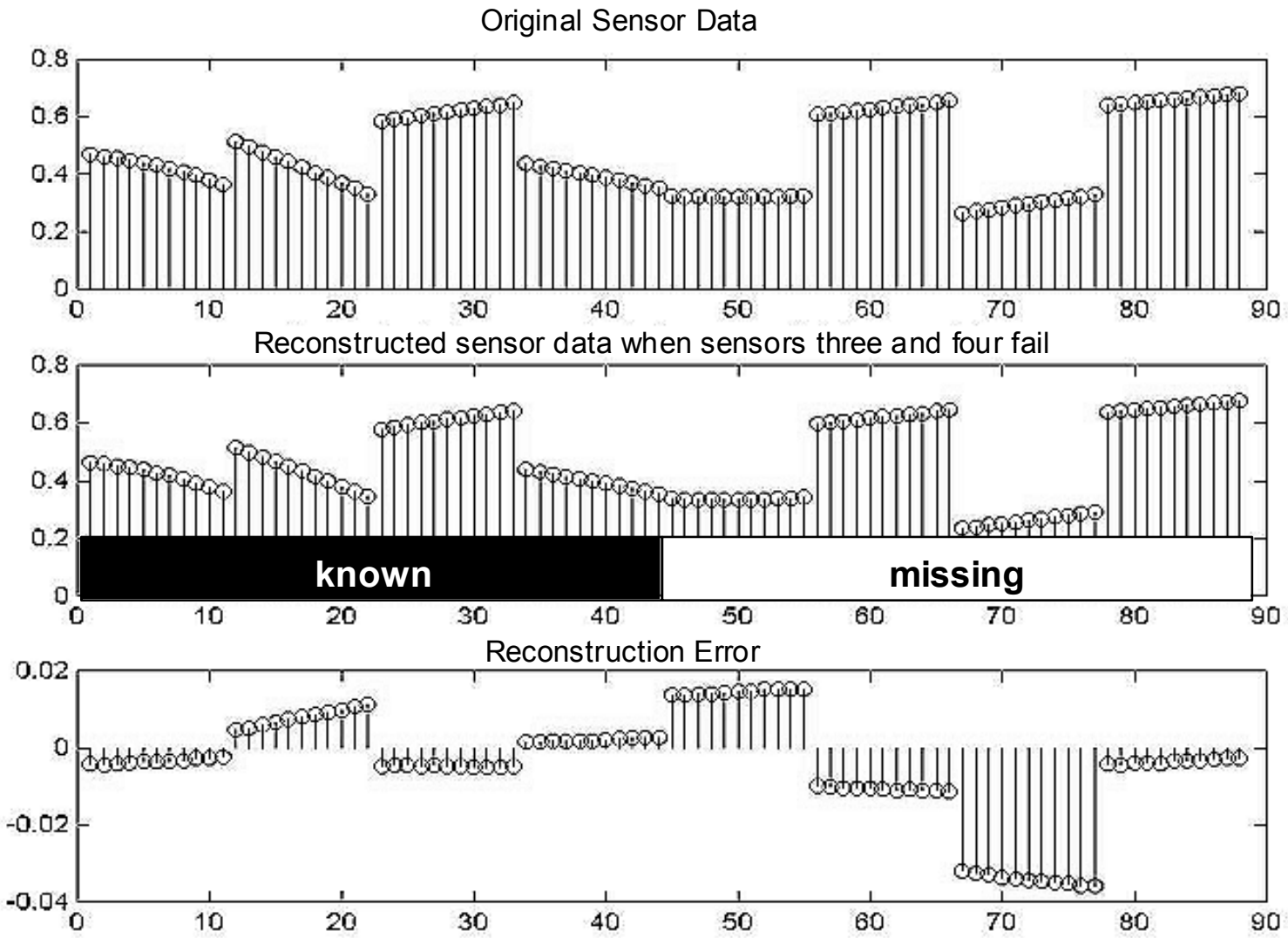

Figure 65: Restoration of two lost vibration sensors corresponding to 44 points of data. 
performance of MISED will degrade, consistent with the results of Oh et al. [5] who show effects of uncertainty are magnified as the number of known sensors diminish.

\section{ACKNOWLEDGEMENTS}

This work is supported by the National Science Foundation and The Boeing Phantom Works at The Boeing Company.

\section{REFERENCES}

1. Sreeram Narayanan, R.J. Marks II, John L. Vian, J.J. Choi, M.A. El-Sharkawi \& Benjamin B. Thompson, "Missing Sensor Data Restoration Using AutoAssociative Regression Machines, Proceedings of the
International Joint Conference on Neural Networks, (May, 2002)

2. S. Oh, R.J. Marks II and D. Sarr, "Homogeneous alternating projection neural networks", Neurocomputing, volume 3, pp. 69-95 (1991).

3. Benjamin B Thompson, Robert J Marks II , Jai J Choi, Mohamed A El-Sharkawi, " Implicit Learning in Autoencoder Novelty Assessment", International Joint Conference on Neural Networks, 2002 IEEE World Congress on Computational Intelligence, May 2002

4. R.D. Reed \& R.J. Marks II, Neural Smithing, (MIT Press, 1999).

5. S. Oh and R.J. Marks II, "Noise sensitivity of projection neural networks", Proc. 1989 IEEE International Symposium on Circuits and Systems, pp.2201-2204, 9-11 May 1989, Portland. 